\title{
CAPÍTULO 19: BIOINSETICIDAS OBTIDOS POR Bacillus thuringiensis MICROENCAPSULADOS: UMA REVISÃO
}

\section{CHAPTER 19: BIOINSECTICIDES OBTAINED BY MICROENCAPSULATED Bacillus thuringiensis: A REVIEW}

\author{
Maria Clara do Nascimento ${ }^{1}$; Marcia Nieves Carneiro da Cunha ${ }^{2}$; Juanize Matias da Silva Batista ${ }^{3}$ \\ Thiago Pajeú Nascimento ${ }^{4}$; Ana Lúcia Figueiredo Porto ${ }^{5}$
}

\begin{abstract}
Resumo
Os insetos compõem o maior grupo do Reino Animal e são extremamente importantes para o equilíbrio ecológico da natureza, muitos são até explorados comercialmente pelos humanos devido à geração de vários produtos, como por exemplo o mel. Apesar de tal positividade, alguns insetos são considerados "vilões" por causarem danos à agricultura e por propagarem diversas doenças. Com isso é vasto o número de pesquisas a fim de produzir inseticidas bastante eficientes, menos danosos ao meio ambiente e a saúde humana, e que tenham um baixo custo de produção. A fonte que mais apresenta essas vantagens é a microbiana, na qual se destaca a bactéria Bacillus thuringiensis $(\mathrm{Bt})$ devido às inúmeras pesquisas e produtos já realizados com sucesso. Outro fator que atende a tais necessidades é a técnica de microencapsulação, que visa melhorar pontos como o efeito, proteção e manipulação dos inseticidas. Por isso, o objetivo desse trabalho é descrever os três pontos supracitados (inseticidas, Bt e microencapsulação) e discutir pesquisas referentes a agentes inseticidas produzidos por $\mathrm{Bt}$ e que sejam microencapsulados. Os trabalhos aqui apresentados demonstram que a Bt é ótima produtora de compostos secundários com ação inseticida e que a microencapsulação de fato potencializa os efeitos dos produtos.
\end{abstract}

Palavras-Chave: Biotecnologia, Microrganismos, Insetos, Revestimento de ingredientes ativos.

\begin{abstract}
Insects make up the largest group in the Animal Kingdom and are extremely important for the ecological balance of nature, many are even commercially exploited by humans due to the generation of various products, such as honey. Despite such positivity, some insects are considered "villains" for causing damage to agriculture and for spreading various diseases. With this, the number of researches is vast in order to produce very efficient insecticides, less harmful to the environment and human health, and that have a low production cost. The source that most presents these advantages is the microbial, in which the bacterium Bacillus thuringiensis $(\mathrm{Bt})$ stands out due to the numerous researches and products already carried out with success. Another factor that meets these needs is the microencapsulation technique, which aims to improve points such as the effect, protection and manipulation of

\footnotetext{
${ }^{1}$ Graduanda em Bacharelado em Ciências Biológicas, Universidade Federal Rural de Pernambuco, mclaranaascimento@hotmail.com

Pós Doutoranda, Universidade Federal Rural de Pernambuco, marcianieves@yahoo.com.br

3 Pós Doutoranda, Universidade Federal Rural de Pernambuco, juanizematias@yahoo.com.br

${ }^{4}$ Pós Doutorando, Universidade Federal Rural de Pernambuco, thiago_pajeu@ hotmail.com

${ }^{5}$ Professora Titular, Universidade Federal Rural de Pernambuco, analuporto@yahoo.com.br
} 
insecticides. Therefore, the objective of this work is to describe the three points mentioned above (insecticides, Bt and microencapsulation) and discuss research related to insecticidal agents produced by $\mathrm{Bt}$ and that are microencapsulated. The works presented here demonstrate that $\mathrm{Bt}$ is an excellent producer of secondary compounds with insecticidal action and that microencapsulation actually enhances the effects of the products.

Keywords: Biotechnology, Microorganisms, Insects, Coating of active ingredients.

\section{Introdução}

A Classe Insecta é a mais numerosa e diversificada do Filo Arthropoda, os insetos são invertebrados que tiveram um enorme sucesso evolutivo devido algumas adaptações como os apêndices articulados e a capacidade de voo (BUZZI, 2013). São animais que estão presentes em todos os ambientes e possuem grande importância ecológica e econômica por realizar importantes funções como, manter o equilíbrio de cadeias alimentares, polinização e produção de produtos comercializados pelo homem como a seda e o mel (PASUPULETI; ARIGELA, 2020; RADER et al., 2016). Os insetos também são utilizados em processos criminais quando se aplica a entomologia forense, onde a partir da espécie de inseto e o estágio do seu desenvolvimento sobre um determinado cadáver pode determinar o tempo de morte do indivíduo (BRUNDAGE; BYRD, 2016). Alguns insetos são danosos à saúde humana por serem vetores ou causadores das arboviroses, como a dengue por exemplo, outros insetos são considerados pragas agrícolas quando nocivos a plantas e produtos vegetais. Tais problemáticas levam a buscas por produtos que sejam utilizados contra os insetos, ou seja, levam aos inseticidas (CAMARA, 2016; SAWICKA; EGBUNA, 2020).

Os produtos utilizados no controle ou extermínio total dos insetos podem ser produzidos a partir de compostos orgânicos ou inorgânicos que sejam de origem sintética ou natural. Bactérias, vírus, fungos e nematoides são fontes naturais de compostos tóxicos aos insetos que geram produtos vantajosos em comparação com os sintéticos devido a diversas vantagens como a baixa taxa de toxicidade a saúde humana, ao meio ambiente e espécies não alvo, e o baixo custo de produção, por exemplo (NETO et al., 2017). O gênero Bacillus envolve bactérias gram-positivas produtoras de endósporos que podem ser encontradas em variados substratos e que são muito utilizadas na produção de pesticidas. A espécie Bacillus thuringiensis $(\mathrm{Bt})$ é produtora de $\delta$-endotoxinas, proteínas com morfologia de cristais conhecidas como proteína Cry, composto que possui atividade entomopatogênica contra diversos insetos, sendo esse grupo um dos principais presentes o mercado de bioinseticidas (GALZER; FILHO, 2016). 
Ultimamente os artefatos utilizados no controle de insetos estão sendo submetidos à técnica de microencapsulação, procedimento no qual uma camada extra pode ser utilizada afim de melhorar vários aspectos desde a produção até a utilização do inseticida, como potencializar o efeito do produto, assegurar boas condições de armazenamento, garantir que o inseticida seja liberado apenas no local exato e pelo tempo desejado, assim diminuindo o tempo de exposição e eventual toxicidade as pessoas que o manipulam e ao meio ambiente, e visando vários outros proveitos (YUSOP et al., 2017).

Contudo, o objetivo dessa revisão narrativa é descrever os principais tópicos que envolvem os bioinseticidas microencapsulados oriundos da bactéria Bacillus thuringiensis, e dissertar algumas pesquisas realizadas nos últimos 5 anos que envolvem esse tema.

\section{Desenvolvimento}

\subsection{Inseticidas}

Devido aos problemas causados por alguns insetos como a proliferação de doenças tal como a dengue, malária, doença de Chagas e leishmaniose, e os inúmeros danos a plantações, como por exemplo a interferência no crescimento das plantas, desvalorização de frutos, morte ou até mesmo a aniquilação total de colheitas, é incessante a busca por produtos que atuem no controle ou erradicação total desses insetos (CHAO et al., 2020; SAEED et al., 2020; SENAPATI et al., 2019; ZHANG et al., 2020). Todo composto capaz de atingir o ovo, a larva ou o inseto já adulto é denominado inseticida, esses são frequentemente utilizados por várias esferas, desde indústrias a uso doméstico (WOJCIECHOWSKA et al., 2016; ZHANG et al., 2020).

A penetração dos inseticidas no corpo do inseto consiste em como ou por onde ele atinge o inseto, assim eles podem ser classificados em: inseticidas de ingestão quando penetram no organismo alvo via oral e são absorvidos pelo intestino; inseticidas de contato são os que entram em contato com o tegumento; os inseticidas de fumigação invadem o organismo através das vias respiratórias; ao agir através da ação translaminar o modo de ação do inseticida é definido como de profundidade; há ainda aqueles que translocam pelo sistema vascular das plantas até atingirem o inseto, os inseticidas sistêmicos (ATHANASSIOU; ARTHUR, 2018).

Os inseticidas podem ser realocados em vários grupos de acordo com o seu sítio de ação, eles podem atuar sobre o sistemas nervoso e muscular, intestino, sistema respiratório, crescimento e desenvolvimento ou podem até ter local de ação inespecífico/desconhecido. 
$\mathrm{Na}$ Tabela 1 estão as classificações de alguns grupos de inseticidas de acordo com sua fisiologia alvo (SPARKS et al., 2020).

Tabela 1. Grupos de inseticidas de acordo com seu sítio de ação.

\begin{tabular}{|c|c|}
\hline Fisiologia Alvo & Grupo \\
\hline \multirow{4}{*}{ Músculo e Nervo } & Inibidores de Acetilcolinesterase \\
\hline & Antagonistas dos canais de cloro regulados por GABA \\
\hline & Moduladores dos canais de sódio \\
\hline & $\begin{array}{l}\text { Bloqueadores } \quad \text { dos } \quad \text { receptores nicotínicos } \\
\text { acetilcolina }\end{array}$ \\
\hline \multirow[b]{2}{*}{ Respiração } & Inibidores da ATP sintase mitocondrial \\
\hline & $\begin{array}{l}\text { Desacopladores da fosforilação } \text { oxidativa } \\
\text { interrupção do gradiente de prótons }\end{array}$ \\
\hline \multirow[t]{2}{*}{ Intestino Médio } & $\begin{array}{l}\text { Inibidores de transporte de elétrons, complexo mitocondrial I, II, } \\
\text { III e IV }\end{array}$ \\
\hline & Disruptores microbianos do intestino dos insetos \\
\hline \multirow{5}{*}{ Crescimento e Desenvolvimento } & Disruptores virais da membrana peritrófica do intestino médio \\
\hline & Simuladores do hormônio juvenil \\
\hline & Inibidores de crescimentro de ácaros \\
\hline & Inibidores da biosíntese de quitina, tipos 0 e 1 \\
\hline & Agonistas do receptor de ecdisona \\
\hline
\end{tabular}

Fonte: Adaptado de Sparks et al. (2020).

Estudos toxicológicos agudos levam a classificação toxicológica dos inseticidas que serve para distinguir os inseticidas mais perigosos dos menos danosos à saúde humana. A Organização Mundial da Saúde (OMS) (2020) classifica a toxicidade do inseticida com base na dose letal média aguda ingerida via oral e dérmica de $50 \mathrm{mg} / \mathrm{kg}$ (DL50) em estudos realizados com ratos, assim os inseticidas e vários outros produtos químicos são divididos em cinco classes:

- Os produtos inseridos na Classe Ia são categorizados como extremamente perigoso quando a DL50 atinge valores $<5 \mathrm{mg} / \mathrm{kg}$ via oral e $<50 \mathrm{mg} / \mathrm{kg}$ via dérmica;

- Quando a DL50 fica entre $5-50 \mathrm{mg} / \mathrm{kg}$ quando ingerido via oral e entre $50-200$ $\mathrm{mg} / \mathrm{kg}$ por via dérmica o produto é considerado altamente perigoso e denominados como Classe Ib;

- $\quad$ Aqueles moderadamente perigosos estão na Classe II que inclui os que atingem $\mathrm{DL}_{50}$ de 50 a $2000 \mathrm{mg} / \mathrm{kg}$ para ambas as vias;

- $\quad$ A Classe III reúne a categoria dos ligeiramente perigosos, que são os de DL50 
oral/dérmica $<2000 \mathrm{mg} / \mathrm{kg}$;

- $\quad$ Aqueles que não são provável que apresentem risco agudo (DL50 $\geq 5000 \mathrm{mg} / \mathrm{kg}$ ) estão dentro da Classe U.

A Resolução da Diretoria Colegiada - RDC no 294, de 29 de julho de 2019 da Agência Nacional de Vigilância Sanitária (ANVISA) define a classificação dos agrotóxicos, afins e preservativos de madeira no Brasil. A mesma assim como a classificação da OMS é baseada na toxicidade aguda da dose letal média, porém, além das vias oral e dérmica insere a via inalatória que compreende aos gases, vapores, produtos sólidos e líquidos (PSeL). Além disso, a ANVISA determina que os agrotóxicos tenham cores pré-definidas em sua rotulagem de acordo com sua posição na classe toxicológica como pode ser visto na Tabela 2. A classe 1 engloba os agrotóxicos extremamente tóxicos, na classe 2 encontram-se os altamente tóxicos, aqueles considerados moderadamente tóxicos estão na classe 3 , os poucos tóxicos estão na classe 4 e os que são improváveis de causar danos agudos estão na classe 5 (BRASIL, 2019).

Tabela 2. Classes Toxicológicas de acordo com a RDC nº 294, de 29 de julho de 2019.

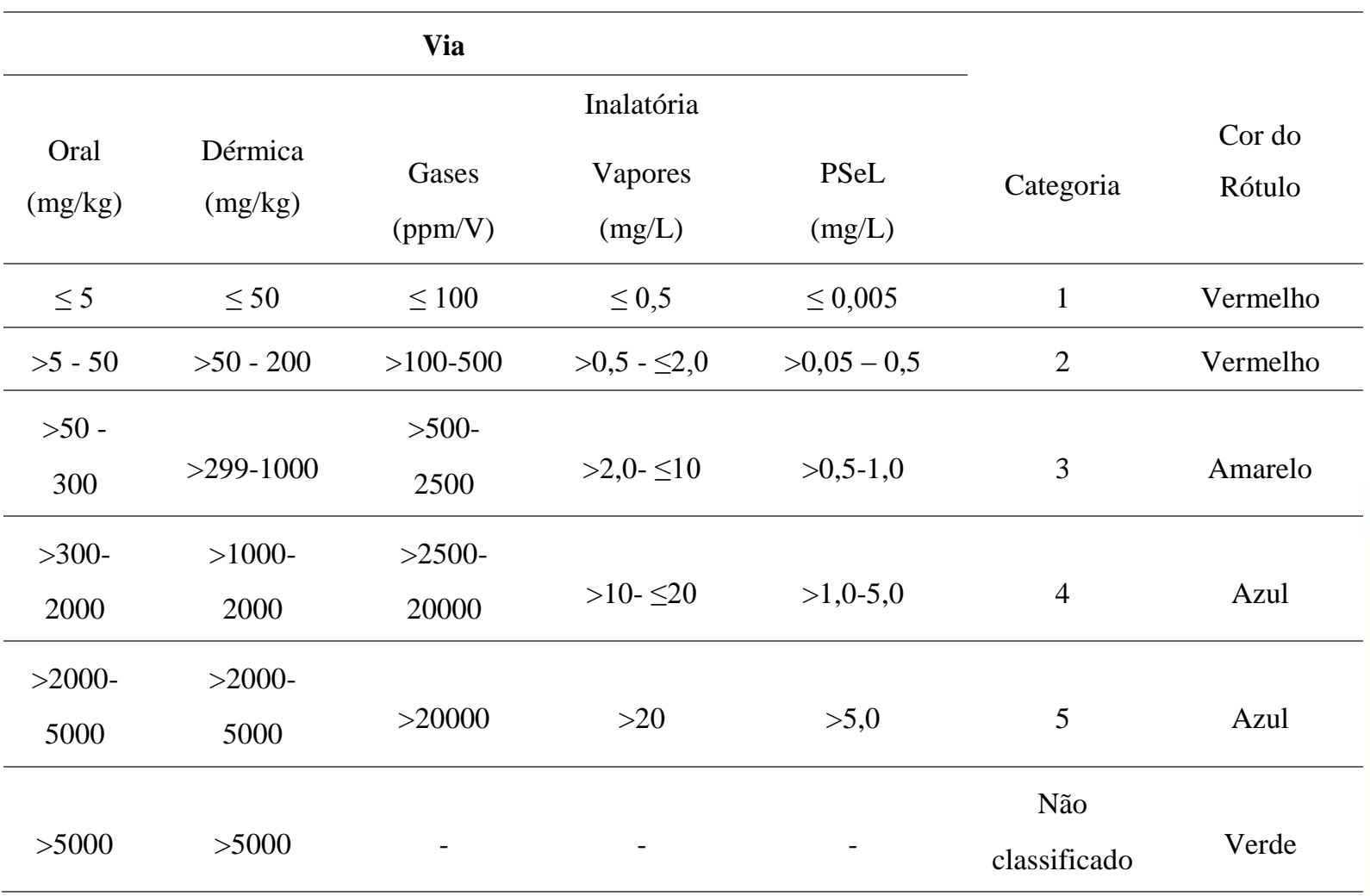

Fonte: Adaptado da ANVISA (2019), apenas para fins ilustrativos.

De acordo com sua fonte, os produtos utilizados no controle ou extermínio de insetos podem ser classificados como inseticidas químicos ou naturais. Os químicos são aqueles que 
sua elaboração é realizada em laboratório utilizando materiais sintéticos e os inorgânicos (GUPTA et al., 2019). Os inseticidas de fontes naturais, ou seja, de origem biológica são os que possuem compostos extraídos de algum vegetal, animal ou microrganismo (PAULRAJ et al., 2016). Existe também a organização desses produtos em gerações. A primeira geração de inseticidas compreende aos inorgânicos como o enxofre e o arsênico, alguns orgânicos de origem vegetal como a nicotina e os organominerais (ATHANASSIOU; ARTHUR, 2018). O início da fabricação de inseticidas sintéticos entre o final do século XIX e início do século XX marcou a segunda geração desses produtos, sendo todos eles de largo espectro, ou seja, não atingia apenas o seu organismo alvo. Os produtos químicos ou naturais que afetam especificamente o crescimento e desenvolvimento dos insetos foram classificados como inseticidas de terceira geração (SOUSA et al., 2020).

Dependendo sua composição química os inseticidas podem ser divididos em dois grupos, os orgânicos, aqueles que possuem carbono em sua estrutura, e os inorgânicos, aqueles que não possuem carbono (ASHITHA; MATHEW, 2020). Os compostos inorgânicos eram os mais usados antigamente, porém devido a sua alta toxicidade aos grupos diferentes dos insetos, inclusive os humanos, eles foram caindo em desuso. Neste grupo encontram-se o chumbo, sulfatos, carbonatos, arsênico, alumínio, boro, cobre e vários outros compostos. $\mathrm{O}$ enxofre e o fosfina são os inseticidas inorgânicos mais utilizados ultimamente sendo eles utilizados contra ácaros e formigas, respectivamente (DAVIS, 2017; SARWAR, 2016). Os inseticidas sintéticos e naturais estão dentro do grande grupo de inseticidas orgânicos. Dentre os sintéticos estão: os organoclorados que possuem $\mathrm{C}, \mathrm{Cl}$ e $\mathrm{H}$ em sua estrutura, muito persistentes no ambiente e nocivos ao homem causando diversas patologias, o dicloro difenil tricloroetano (DDT) (Figura 1) é um exemplo desse tipo de inseticida (JAYARAJ et al., 2016; PARADA et al., 2016); os organofosforados (Figura 2) incluem o ácido fosfórico e seus derivados com espectro de ação variável (OCK et al., 2020); na classe dos carbamatos (Figura 3) estão os inibidores da acetilcolinesterase elaborados a partir de alcaloides (JANKOWSKA, 2019); piretroides são os análogos sintéticos das piretrinas naturais, sua estrutura química é caracterizada pela presença de um anel com três carbonos, grupo carboxílico e um grupo vinil, a deltametrina (Figura 4) é um inseticida piretroide muito vendido no Brasil (ENSLEY, 2018); os compostos derivados e melhorados da nicotina formal a classe dos neonicotinóides, o primeiro a ser comercializado em meados anos 90 foi o imidaclopride (Figura 5) (MATSUDA et al., 2020); amitraz, fenilpirazóis (Figura 6) são outras classes químicas dos inseticidas (GUPTA; ANADÓN, 2018; KITA et al., 2017). 
Figura 1. Estrutura do DDT.<smiles>Clc1ccc(C(c2ccc(Cl)cc2)C(Cl)(Cl)Cl)cc1</smiles>

Figura 3. Estrutura do carbamato.<smiles>[R]OC(=O)N([R])[R]</smiles>

Fonte: Adaptado de Sousa (2020).

Figura 5. Estrutura do imidaclopride.

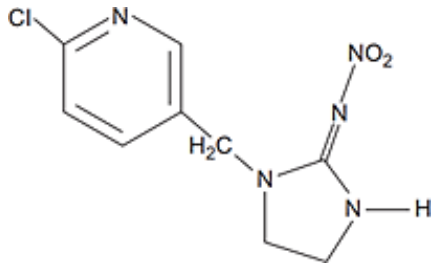

Fonte: Adaptado de Sousa (2020).
Figura 2. Estrutura do organofosforado.<smiles>[R]OP([R20])([R20])=O</smiles>

Fonte: Adaptado de Sousa (2020).

Figura 4. Estrutura do deltametrina.<smiles>CC1(C)C(C=C(Br)Br)C1C(=O)OC(C#N)c1cccc(Oc2ccccc2)c1</smiles>

Fonte: Adaptado de Sousa (2020).

Figura 6. Estrutura do fipronil

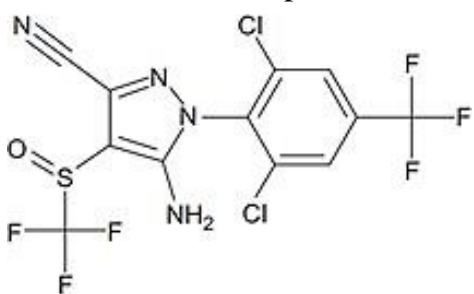

Fonte: Adaptado de Gupta e Anadón (2018).

Quando extraídos de animais, microrganismos, minerais ou plantas os inseticidas são ditos como naturais. Esse tipo de inseticida é mais interessante por causar menos danos à saúde humana e ao ambiente, e por serem biodegradáveis. Algumas plantas são produtoras de compostos secundários tóxicos aos insetos, como por exemplo a Tanacetum cinerariifolium na qual a partir de suas flores secas é extraído o piretro, composto que atinge o sistema nervoso dos insetos levando-os a morte. A azadiractina é um bioativo que pode ser obtido das sementes de Azadirachta indica, conhecida como Nim, e são utilizados na produção de inseticidas naturais. Vários óleos essenciais de outras plantas também são utilizados no controle biológico de insetos (IKBAL; PAVELA, 2019; ISMAN, 2020). Os estudos envolvendo microrganismos entomopatogênicos estimularam os pesquisadores a desenvolver produtos que agissem no controle de insetos, os metabólicos produzidos por esses indivíduos são altamente tóxicos para os insetos e inofensivos a saúde de outros animais e a natureza. Um dos motivos também do sucesso dos inseticidas microbianos é a sua facilidade de fabricação e alta produtividade por baixo custo, já que os bioprocessos envolvidos são bastantes simples e baratos. Metapremium, BoveMax EC®, CartuchoVIT e 
Bt-horus são alguns inseticidas microbianos já presentes no comércio (NAWAZ et al., 2016). Alguns dos microrganismos utilizados na produção de bioinseticidas são: Hirsutella sp., Phytophthora sp., Colletotrichum sp., Trichoderma sp., Metarhizium sp., Beauveria sp., Sporothrix sp., Aschersonia sp., Aspergillus sp., Enthomophthora sp., Penicillium sp., Nosema sp., Baculoviridae, Bacillus sp. e Pseudomonas sp.

\subsection{Bacillus thuringiensis}

Um dos principais microrganismos utilizados como agente bioinseticida é a bactéria do solo Bacillus thuringiensis (Bt). Essas bactérias são bioinseticidas seguros, biodegradáveis e de alto potencial, com elevada taxa de letalidade contra os insetos agrícolas (DEVI et al., 2019). Seu potencial entomopatogênico se deve a capacidade de sintetizar vários cristais proteicos durante a esporulação conhecidos como delta-endotoxinas. As delta-endotoxinas de $B$. thuringiensis são produzidas em diversas morfologias cristalinas como bipiramidais, cuboidais, esféricos e romboidais, onde cada tipo de cristal possui atividade inseticida apenas para uma ordem de inseto, e isso faz com que bioinseticidas formulados a base de Bt sejam ecologicamente seguros por não atingirem populações de insetos benéficos, outros invertebrados e vertebrados (FREIRE DA SILVA et al., 2020). Após a ingestão, estas toxinas são ativadas pela ação das enzimas digestivas que atuam em $\mathrm{pH}$ alcalino, ligam-se a receptores específicos nas células epiteliais do intestino médio e induzem poros na membrana que conduzem, habitualmente, a septicemia e morte de insetos (HERRERO et al., 2016).

\subsection{Microencapsulação}

Entretanto, a maioria dos entomopatógenos possuem algumas limitações quanto a sua utilização no campo, pois são suscetíveis à rápida degradação ambiental devido à radiação UV, calor, dessecação, pH do substrato e competição microbiana. Para garantir a eficácia, manipulação proveitosa, melhores condições de armazenamento, segurança e fácil aplicação os inseticidas precisam sofrer alteração de sua forma bruta para a utilizável, esse processo é denominado formulação. A formulação é composta pelo ingrediente ativo (substância que realmente controla a praga) e ingredientes inertes como surfactantes, um veículo e outros ingredientes como os corantes. Os ingredientes inertes são aditivos que visam a melhoria da manipulação do produto, aumenta sua toxicidade, proteção contra fatores ambientais, etc. Os tipos de formulações mais utilizadas são: pó seco, solúvel ou molhável; soluções concentradas; emulsões e concentrados emulsionável; suspensões concentradas; aerossol; 
gases; granulados; pasta; adesivos e microencapsulado (WATANABE et al., 2016).

A microencapsulação é uma técnica em que micropartículas são envolvidas por uma camada a fim de protegê-las ou melhorar seu desempenho. Esse método visa proteger o material encapsulado de vários fatores como $\mathrm{pH}$, temperatura e reações químicas indesejadas com outras materiais e também melhora a estabilidade e a viabilidade do produto. Micropartículas são partículas esféricas sólidas, liquidas ou gasosas que possuem de 1 a1000 $\mu \mathrm{m}$. Em relação a sua estrutura as micropartículas podem ser classificadas em um tipo matricial em que a substância pode estar na superfície ou no interior denominado microesferas, e em microcápsulas onde a substância encontra-se no núcleo e envolvida por uma camada construída durante o processo de microencapsulação, esses dois tipos de micropartículas podem ser observados na Figura 7 (SUGANYA; ANURADHA, 2017).

Figura 7. Representação de uma microesfera (A) e Representação de uma micropartícula (B).

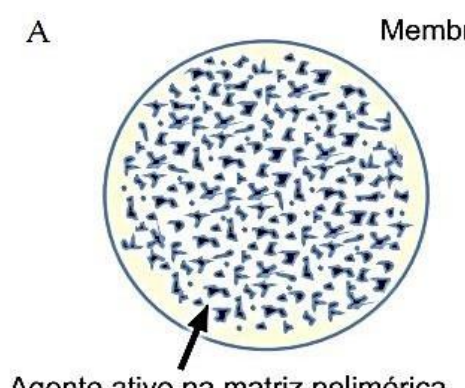

Agente ativo na matriz polimérica

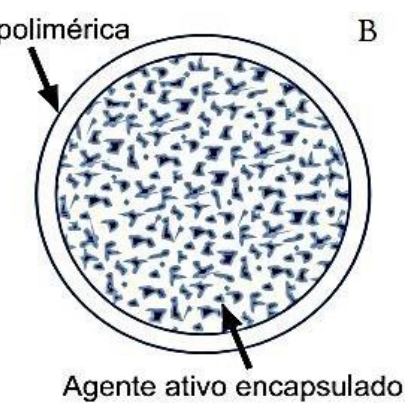

Agente ativo encapsulado

Fonte: Adaptado de Suave et al. (2006).

Os compostos utilizados no envolvimento das micropartículas são definidos agentes encapsulantes. Eles podem ser obtidos de fontes naturais, sintéticas ou semissintéticas. Cera, sacarose e gelatina são alguns dos tipos naturais. Os sintéticos envolvem polímeros e os semissintéticos acetato de celulose, nitrato de celulose e outros. O sucesso dos compostos bioativos encapsulados depende do tipo de substância que será utilizada na sua microencapsulação, o que é definido por suas características químicas e físicas, seu método de microencapsulamento e seu tipo de aplicação. A viscosidade, a capacidade de absorver água, capacidade de proteção, fácil manuseio durante o processo, boas condições de liberação da micropartícula e baixo custo são alguns pontos que devem ser estudados durante a escolha da substância que será utilizada na encapsulação (BHATIA, 2020; SONAWANE, 2020).

A maneira como ocorre a liberação do bioativo presente no núcleo da microcápsula é ponto crucial no sucesso da ação da micropartícula em seu uso final. Geralmente a escolha 
da técnica da encapsulação foca o controle da excreção da substância envolvida tendo a finalidade de definir o modo de como ela será liberada, os locais onde o bioativo precisa ser preservado ou liberado para começar agir, o tempo em que ele levará para começar a ser liberado e a duração da mesma (CASTRO et al., 2017). A eliminação do núcleo pode ocorrer de maneira mecânica quando algo entra em atrito com o microencapsulado e rompe o revestimento da partícula, por dissolução do encapsulado em solvente de interesse, dependendo da temperatura que muda o estado da matéria ou por meio de alterações químicas. O material utilizado na envoltura da micropartícula e o modo de liberação definido para tal irão definir a velocidade e a quantidade do produto liberado, o que também depende de qual será o uso da substância (CANDIAGO et al., 2018).

As metodologias de microencapsulação irão depender das propriedades do bioativo, principalmente as química e física, devido as interações da micropartícula com o material escolhido para o envelopamento e o seu tamanho. O número de técnicas já reconhecidas por patente é numeroso e vem crescendo cada vez mais, todos os métodos existentes podem ser em três grandes grupos: químico, físico e físico-químico. Alguns dos métodos físicos são: a liofilização, processo onde um material líquido congelado é desidratado por sublimação (PISANO et al., 2019); o spray chilling, método de congelamento por atomização (YIN; CADWALLADER, 2018); e o leito fluidizado, técnica na qual um sólido com menos que $100 \mu \mathrm{m}$ é transformado liquido por algum gás ou líquido quente e seco (ESTEVINHO; ROCHA, 2018). Entre os métodos químicos podem ser encontrados a polimerização interfacial, nessa técnica se usa uma emulsão água/óleo para polimerizar os monômeros

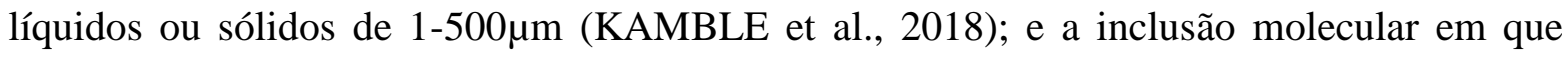
normalmente líquidos são encapsulados por $\beta$-ciclodextrina (OZKAN et al., 2018). Entre os métodos físico-químicos mais utilizados estão: a coacervação, onde micropartículas menores que $500 \mu \mathrm{m}$ são separadas devido a formação de três fases imisturáveis seguida da deposição e endurecimento do material encapsulante sobre a micropartícula, quando se utiliza só um polímero a coacervação é denominada simples, caso sejam utilizados mais de um passa a ser chamada de coacervação complexa e a emulsificação variando a utilização de água e óleo seguida de evaporização do solvente utilizado; além delas existem outras técnicas como a pulverização em agente formador de reticulação e envolvimento lipossômico (GUMFEKAR, 2020; TIMILSENA et al., 2019).

A aplicação da microencapsulação abrange diversas áreas como o ramo farmacêutico, onde o método é utilizado para melhorar o sabor, liberação, odor e redução de 
irritações dos medicamentos. Paracetamol, Diclofenaco e Ácido acetilsalicílico são alguns medicamentos que obtiveram sucesso ao fazer uso da microencapsulação (AKEL et al., 2017; ANEES et al., 2018; SAKPAKDEEJAROEN et al., 2020); na área alimentícia o uso da técnica beneficia a proteção do produto a condições externas que possa diminuir suas qualidades como o gosto e aroma, como também aumenta o tempo de armazenamento. No ramo alimentício muito usada em óleo de peixe, corantes, acidulantes e em enzimas como as de Lactobacillus sp (BAKRY et al., 2016; MU et al., 2018). Além disso, a microencapsulação é utilizada em cosméticos e produtos agrícolas (NANDY et al., 2020; ZHANG et al., 2019).

O uso da técnica de microencapsulação em pesticidas alveja alcançar a proteção do

produto contra fatores ambientais externo como a chuva e o sol, influenciam a biodisponibilidade e na seletividade da espécie alvo (RAJAN et al., 2020). Uma das maiores vantagens da microencapsulação dos pesticidas é a segurança que repassa para quem trabalha aplicando os mesmos e ao meio ambiente devido ao fato de controlar o tempo de exposição. Múltiplos pesticidas já foram encapsulados como o herbicida trifluralina, óleos de Citrus sp., decadienoato e os oriundos de Bacillus thuringiensis (CHI et al., 2020; DE ARAÚJO et al., 2020).

\subsection{Discussão}

Após pesquisas sobre bioinseticidas produzidos pelo gênero Bacillus foi verificado que, Khorramvatan et al. (2017) microencapsulou B. thuringiensis subsp. kurstaki KD-2 (BtKD2) utilizando como agente encapsulante alginato de sódio através de gelificação por emulsão água/óleo. O processo seguiu um planejamento que variava a concentração cloreto de cálcio $(0,1$ e $0,3 \mathrm{M})$, alginato de sódio $(2$ e $3 \%$ p/p) e a velocidade de agitação nas variáveis respostas: viabilidade dos esporos, mortalidade e diâmetro. Os autores observaram a morfologia das microcápsulas por microscopia óptica e o efeito da radiação ultravioleta (UVB) sobre elas. O inseticida microencapsulado foi exposto a ínstares larvais de Ephestia kuehniella Zeller e a partir disso a mortalidade foi estudada durante 7 dias. O ensaio que apresentou a maior viabilidade dos esporos (79\%) foi o com concentração 2,2\% (p/p) de alginato de sódio; $0,26 \mathrm{M}$ de $\mathrm{CaCl}_{2}$ e velocidade de agitação de $1898 \mathrm{rpm}$. O produto atingiu cerca de $93 \%$ de mortalidade sobre Ephestia kuehniella. E diâmetro de até $23 \mu$. Após estudos estatísticos foi visto que, a combinação entre o alginato e $\mathrm{CaCl}_{2}$ influenciaram no aumento do diâmetro da micropartícula. A concentração do polímero teve forte influência 
sobre a viabilidade dos esporos. As melhores condições para o microencapsulamento foram alginato de sódio 2,15\%, $\mathrm{CaCl} 2$ 0,24 M e velocidade $1606 \mathrm{rpm}$. A viabilidade, mortalidade e diâmetro dos esporos foram de $68 \%, 85 \%$ e $9 \mu$ respectivamente. Sob UVB a viabilidade dos esporos caiu para $40 \%$ e a mortalidade para 50\%. Os autores constataram que tal procedimento é viável na microencapsulação de Bt-KD2.

He e colaboradores (2017) realizaram a microencapsulação de proteínas de cristais (Cry1Ac) produzidas por Bacillus thuringiensis HD73 (Bt-HD73). Os agentes encapsulantes utilizados foram quitosana e alginato, em cada microcápsula foram construídas 10 camadas a partir de um ciclo de deposição de quitosana-alginato. Foi testada a toxina normal sem ter passado pelo processo de microencapsulação e a que foi encapsulada. Os autores detalharam morfologicamente as microcápsulas, a liberação da toxina dependendo do $\mathrm{pH}$ e sua resistência sob estresses ambientais como, altas temperaturas, dessecação e raios ultravioleta. O produto foi testado contra a mariposa Ostrinia furnacalis. A microscopia realizada para observação do tamanho revelou que a microcápsula tinha cerca de $0,6 \times 1,2 \mu \mathrm{m}$. Foi detectado que a maior liberação das proteínas foi ocorreu quando o produto estava em $\mathrm{pH} \geq 9,0$. Quando expostas a temperatura ambiente por mais de 10 dias, as proteínas perderam suas propriedades físico- químicas, porém sob temperatura de $50^{\circ} \mathrm{C}$ essas propriedades foram mantidas. A eficácia da proteção da microencapsulação também foi comprovada quando o produto foi submetido a dessecação. A exposição a UVB após o estado de extrema secura por mais de 3 - 6 horas diminuiu tal proteção. A mortalidade das larvas de $O$. furnacalis variou de 70 a $50 \%$ em condições normais, após o produto ter passado por estresse ambiental houve uma queda na mortalidade das larvas. A toxicidade dos inseticidas não microencapsulados e microencapsulados foram equivalentes.

As proteínas produzidas por Bacillus thuringiensis var. kurstaki HD-1 (Bt-HD1) foi objeto de estudo da pesquisa de Cortés et al. (2017). Os autores realizaram microencapsulação pelo método de emulsão por inversão utilizando água e óleo de milho. $\mathrm{O}$ produto foi exposto a larvas de Spodoptera frugiperda para ser ingerido via oral. Foram realizadas analises de dois ensaios diferentes, um observou a influência da baixa concentração das proteínas produzidas por Bt-HD1 e no outro o efeito do diâmetro da microcápsula. Após sete dias do início do experimento foi verificada a quantidade de indivíduos mortos e o peso dos sobreviventes. Com os resultados foi observado que a diminuição do diâmetro da microcápsula aumentou a mortalidade da espécie-alvo, microcápsulas de $3,1 \mu \mathrm{m}$ aumentaram mais de $105 \%$, as de $3,2 \mu \mathrm{m}$ fizeram a mortalidade 
variar entre $93-74 \%$, já as com $9,5 \mu \mathrm{m}$ atingiram cerca de $80 \%$ da população de larvas. Com apenas $40 \mathrm{ng} / \mathrm{cm}^{2}$ de concentração das proteínas produzidas por Bacillus thuringiensis var. kurstaki HD-1 a mortalidade chegou em 70\%. Quando expostas a concentração de $10 \mathrm{ng} / \mathrm{cm}^{2}$ as larvas aumentaram de tamanho, o mesmo também ocorreu quando foram disponibilizadas microcápsulas com altas concentrações de proteínas. As microcápsulas preservaram suas características mesmo após serem expostas a raios ultravioleta.

Eski et al. (2019) em seu trabalho isolou uma cepa de B. thuringiensis S13 (Bt-Se13) e a inoculou em biorreator com fermentação em estado líquido contendo farinha de soja, glicose e outros ingredientes durante $72 \mathrm{~h}$ a $30^{\circ} \mathrm{C}$. O extrato junto foi lavado para retirada de enzimas e toxinas indesejáveis e seguiu para microencapsulação utilizando o método de spray drying $\left( \pm 4^{\circ} \mathrm{C}\right)$ seguindo um planejamento de $2^{3}$ variando o tipo de agente encapsulante (amido, soja modificados, maltodextrina DE10 e goma-arábica), a temperatura de entrada $\left(120,140,160\right.$ e $\left.180^{\circ} \mathrm{C}\right)$ e temperatura de saída $\left(60,70,80\right.$ e $\left.90^{\circ} \mathrm{C}\right)$ com uma matriz de 16 ensaios. Para verificar as características do microencapsulado feito em ótimas condições foram verificados a morfologia, o tamanho da micropartícula, a determinação de molhagem do pó, a suspensabilidade, o teor de umidade e a entomopatogenicidade de Bt-Se13 microencapsulado, não miroencapsulado e de um produto comercial frente a larvas de Spodoptera exigua em condições de laboratório e plantio em vaso. A mortalidade foi estudada ao decorrer de 10 dias. Após análises estatísticas os autores verificaram que, sobre a microencapsulação com produzida com Maltodextrin DE10, temperatura de entrada de $180^{\circ} \mathrm{C}$ e temperatura de saída de $90^{\circ} \mathrm{C}$ o tipo de agente encapsulante foi o que mais influenciou; a temperatura de entrada foi a que mais influenciou no encapsulamento contendo goma-arábica, $160^{\circ} \mathrm{C}$ de entrada e $80^{\circ} \mathrm{C}$ de saída; já o microencapsulamento feito a temperatura de $140^{\circ} \mathrm{C}$ na entrada e $70^{\circ} \mathrm{C}$ e na saída amido modificado sofreu influência da temperatura de saída. O estudo de caracterização da micropartícula revelou que medição da micropartícula foi de $13,462 \mu \mathrm{m}$, o tempo de molhagem 25,22 segundos, a suspensabilidade $77,66 \%$, e o teor de umidade 7,29\%. Todos os compostos testados contra S. exigua demonstraram $100 \%$ de mortalidade quando a plicados na concentração de $10^{9} \mathrm{ml}^{-1}$. Nos ensaios feito em laboratório, a toxicidade aguda do Bt-Se13 microencapsulado sob a espécie alvo foi $1.6 \times 10^{4} \mathrm{UFC} \mathrm{ml}^{-1}$, a toxicidade causada pelo Bt- Se13 que não passou pelo processo de microencapsulação foi $1.0 \times 10^{5} \mathrm{ml}^{-1}$ e o produto comercial causou $4.6 \times 10^{4} \mathrm{ml}^{-1}$. No plantio em vaso $93 \%$ das larvas foram mortas por Bt-Se13 microencapsulado e $90 \%$ pelo produto do já disponível no comercio. 


\section{Considerações Finais}

Contudo, é notável a elevada eficácia dos compostos secundários produzidos por Bacillus thuringiensis frente ao combate aos insetos, e que, o microencapsulamento desses bioativos potencializa a sua ação. Isso demonstra a importância da elaboração de revisões como essa acerca do assunto, para que se torne interessante e numerosa a continuação e realização de novas pesquisas para o desenvolvimento de bioinseticidas microencapsulados contendo o Bt como ingrediente ativo.

\section{Referências}

AKEL, H.; MADANI, F.; IBRAHIM, W. Preparation of Paracetamol Microcapsules by Complex Coacervation and Studying the effect of some Factors Influencing Microencapsulation Yield and Efficacy. Research Journal of Pharmacy and Technology, v. 10, n. 10, p. 3271-3275, 2017.

ANEES, M. et al. Formulation and In-Vitro Evaluation of Polymers Blend Based Diclofenac Sodium Microparticles for Sustained Release Drug Delivery. RADS Journal of Pharmacy and Pharmaceutical Sciences, v. 6, n. 3, p. 171-177, 2018.

ASHITHA, A. MATHEW, J. Characteristics and Types of Slow/Controlled Release of Pesticides. In: RAKHIMOL, K. R. Controlled Release of Pesticides for Sustainable Agriculture. 1. ed. Switzerland: Springer, 2020. Cap. 6, p. 141-153.

ATHANASSIOU, C. G.; ARTHUR, F. H. Bacterial Insecticides and Inert Materials. In: ATHANASSIOU, C. G.; ARTHUR, F. H. Recent Advances in Stored Product Protection. 1. ed. Berlin: Springer-Verlag, 2018. cap. 5, p. 83-98.

BAKRY, Amr M. et al. Microencapsulation of oils: A comprehensive review of benefits, techniques, and applications. Comprehensive reviews in food science and food safety, $\mathrm{v}$. 15, n. 1, p. 143-182, 2016.

BHATIA, M. A review on application of encapsulation in agricultural processes. In: SONAWANE, H. S.; BHANVASE, B. A; SIVAKUMAR, S. Encapsulation of Active Molecules and Their Delivery System. India: Elsevier, 2020. cap. 8, p. 131-140.

BRASIL. Agência Nacional de Vigilância Sanitária. Guia $N^{\circ}$ 12/2019 - Versão 2: Guia para elaboração de rótulo e bula de agrotóxicos, afins e preservativos de madeira. Brasília, 2019.

BRASIL. Agência Nacional de Vigilância Sanitária. Resolução da Diretoria Colegiada RDC No 302, de 23 de Agosto de 2019.Diário Oficial da União. Brasília, 28 ago. 2019. Disponível em:http://portal.anvisa.gov.br/documents/10181/2858730/REP_RDC_296_2019.pdf/0a4d94 7 a-1509-40dc-beac-d233e08b4149. Acesso em 08 jun. 2020.

BRUNDAGE, A.; BYRD, J. H. Forensic entomology in animal cruelty cases. Veterinary 
pathology, v. 53, n. 5, p. 898-909, 2016.

CANDIAGO, N. T.; ANSILIERO, R.; GELINSKI, J. L. N. Revisão sobre métodos de microencapsulação. Anuário Pesquisa e Extensão Unoesc Videira, v. 3, p. e17281-e17281, 2018.

CAMARA, T. N. L. Arboviroses emergentes e novos desafios para a saúde pública no Brasil. Revista de Saúde Pública, v. 50, p. 36, 2016.

CHAO, C.; LEONE, J. L.; VIGLIANO, C. A. Chagas disease: Historic perspective. Biochimica et Biophysica Acta (BBA)-Molecular Basis of Disease, v. 1866, n. 5, p. 165689, 2020.

CHI, Y. et al. Microencapsulation of Bacillus megaterium NCT-2 and its effect on remediation of secondary salinization soil. Journal of Microencapsulation, v. 37, n. 2, p. 134-143, 2020.

CORTÉS, J. B. et al. Reducing the microcapsule diameter by micro-emulsion to improve the insecticidal activity of Bacillus thuringiensis encapsulated formulations. Biocontrol Science and Technology, v. 27, n. 1, p. 42-57, 2017.

DAVIS, F. R. Inseticidas, agricultura e antropoceno. Ambiente Global, v. 10, n. 1, p. 114$136,2017$.

DE ARAÚJO, J. S. F. et al. Microencapsulation of sweet orange essential oil (Citrus aurantium var. dulcis) by liophylization using maltodextrin and maltodextrin/gelatin mixtures: Preparation, characterization, antimicrobial and antioxidant activities. International journal of biological macromolecules, v. 143, p. 991-999, 2020.

DEVI, P. S. V.; DURAIMURUGAN, P.; CHANDRIKA, K. S. V. P. Bacillus thuringiensisbased nanopesticides for crop protection. In: Nano-biopesticides today and future perspectives. Academic Press, 2019. p. 249-260.

ENSLEY, S. M. Pyrethrins and Pyrethroids. In: GUPTA, R. C. Veterinary Toxicology: Basic and Clinical Principles. 3. ed. United States: Academic Press, 2018. cap. 39, p. 515520.

ESKI, A.; DEMIRBAĞ, Z.; DEMIR, İ. Microencapsulation of an indigenous isolate of Bacillus thuringiensis by spray drying. Journal of microencapsulation, v. 36, n. 1, p. 1-9, 2019.

ESTEVINHO, B. N.; ROCHA, F. Application of Biopolymers in Microencapsulation Processes. In: GRUMEZESCU, A. M.; HOLBAN, A. M. Biopolymers for Food Design. ed. 20. Cambridge: Academic Press, 2018. cap. 7, p. 191-222.

FREIRE DA SILVA, T. A., et al. Optimization of a culture medium based on forage palm for $\delta$-endotoxin production, Biocatalysis and Agricultural Biotechnology, Volume 27, August 2020, 101664. 
GALZER, E. C. W.; FILHO, W. S. A. Utilização do Bacillus thuringiensis no controle biológico de pragas. Revista Interdisciplinar de Ciência Aplicada, v. 1, n. 1, p. 13-16, 2016.

GUMFEKAR, S. P. Physicochemical characterization techniques in the encapsulation of active molecules. In: SONAWANE, H. S.; BHANVASE, B. A; SIVAKUMAR, S.

Encapsulation of Active Molecules and Their Delivery System. India: Elsevier, 2020. cap. 2, p. 9-22.

GUPTA, C. R.; ANADÓN, A. Fipronil. In: GUPTA, R. C. Veterinary Toxicology: Basic and Clinical Principles. 3. ed. United States: Academic Press, 2018. cap. 39, p. 533 - 538.

GUPTA, R. C. Insecticides. In: GUPTA, R. C. Biomarkers in Toxicology. 2. ed. London: Academic Press, 2019. cap. 26, p. 455-475.

HE, X. et al. Biopolymer microencapsulations of Bacillus thuringiensis crystal preparations for increased stability and resistance to environmental stress. Applied microbiology and biotechnology, v. 101, n. 7, p. 2779-2789, 2017.

HERRERO, S. et al. Susceptibility, mechanisms of response and resistance to Bacillus thuringiensis toxins in Spodoptera spp. Current Opinion in Insect Science, v. 15, p. 89-96, 2016.

IKBAL, C.; PAVELA, R. Essential oils as active ingredients of botanical insecticides against aphids. Journal of Pest Science, p. 1-16, 2019.

ISMAN, Murray B. Botanical insecticides in the twenty-first century-fulfilling their promise?. Annual Review of Entomology, v. 65, p. 233-249, 2020.

JANKOWSKA, M. et al. The unusual action of essential oil component, menthol, in potentiating the effect of the carbamate insecticide, bendiocarb. Pesticide biochemistry and physiology, v. 158, p. 101-111, 2019.

JAYARAJ, R.; MEGHA, P.; SREEDEV, P. Pesticidas organoclorados, seus efeitos tóxicos nos organismos vivos e seu destino no meio ambiente. Toxicologia Interdisciplinar, v. 9, n. 3-4, p. 90-100, 2016.

KAMBLE, V.; SAWANT, M.; MAHANWAR, P. Microencapsulation of cypermethrin via interfacial polymerization for controlled release application. Materials Today: Proceedings, v. 5, n. 10, p. 22621-22629, 2018.

KHORRAMVATAN, S. et al. Optimising microencapsulated formulation stability of Bacillus thuringiensis subsp. kurstaki (Bt-KD2) against ultraviolet condition using response surface methodology. Archives of Phytopathology and Plant Protection, v. 50, n. 5-6, p. 275-285, 2017.

KITA, T. et al. Amitraz and its metabolite differentially activate $\alpha$ - and $\beta$-adrenergic- like octopamine receptors. Pest management science, v. 73, n. 5, p. 984-990, 2017. 
MATSUDA, K.; IHARA, M.; SATTELLE, D. B. Neonicotinoid insecticides: molecular targets, resistance, and toxicity. Annual Review of Pharmacology and Toxicology, v. 60, p. 241-255, 2020.

MU, R. J. et al. Microencapsulation of Lactobacillus acidophilus with konjac glucomannan hydrogel. Food Hydrocolloids, v. 76, p. 42-48, 2018.

NANDY, A. et al. Microencapsulation of retinyl palmitate by melt dispersion for cosmetic application. Journal of Microencapsulation, v. 37, n. 3, p. 205-219, 2020.

NAWAZ, M.; MABUBU, J. I.; HUA, H. Situação atual e avanço dos biopesticidas: pesticidas microbianos e botânicos. J Entomol Zool Stud , v. 4, n. 2, pág. 241-246, 2016.

NETO, G. J. C. et al. Bioprospecção de produtos naturais inseticidas. In: Anais do Congresso Brasileiro de Fitossanidade. 2017.

OCK, J.; KIM, J; CHOI, Y. Organophosphate insecticide exposure and telomere length in US adults. Science of The Total Environment, v. 709, p. 135990, 2020.

OZKAN, G. et al. A review of microencapsulation methods for food antioxidants: Principles, advantages, drawbacks and applications. Food chemistry, v. 272, p. 494-506, 2019.

PARADA J. R. H. et al. Organochlorine insecticides DDT and chlordane in relation to survival following breast cancer. International Journal of Cancer, v. 138, n. 3, p. 565-575, 2016.

PASUPULETI, V. R.; ARIGELA, C. S. Polyphenols and Flavonoids from Honey: A Special Focus on Diabetes. In: KUMAR, D.; SHAHID, M. Natural Materials and Products from Insects: Chemistry and Applications. Switzerland: Springer, 2020. cap. 1, p. 1-20.

PAULRAJ, M. G. Natural Insecticides from Actinomycetes and Other Microbes for Vector Mosquito Control. In: VEER, V. \& GOPALAKRISHNAN, R. Herbal Insecticides, Repellents and Biomedicines: Effectiveness and Commercialization. Índia: Springer, 2016. Cap. 5, p. 85-99.

PISANO, R. et al. Achieving continuous manufacturing in lyophilization: Technologies and approaches. European Journal of Pharmaceutics and Biopharmaceutics, v. 142, p. 265$279,2019$.

RADER, R. et al. Non-bee insects are important contributors to global crop pollination. Proceedings of the National Academy of Sciences, v. 113, n. 1, p. 146-151, 2016.

RAJAN, M. et al. Controlled Release Pesticides as a Route to Sustainable Crop Production. In: RAKHIMOL, K. R. et al. Controlled Release of Pesticides for Sustainable Agriculture. Switzerland: Springer, 2020. p. 111-125.

SAEED, F. et al. Transgenic technologies for efficient insect pest management in crop plants. In: USHA, K.; MALIK, Z. A.; KAMALUDDIN. Transgenic Technology Based Value Addition in Plant Biotechnology, p. 123, 2020. 1 ed. Academic Press: EUA, 2020. Cap 6, p. 123-156. 
SAKPAKDEEJAROEN, I. et al. Development of transferrin-bearing vesicles encapsulating aspirin for cancer therapy. Journal of liposome research, v. 30, n. 2, p. 174-181, 2020.

SARWAR, M. Inseticidas inorgânicos usados em cenários paisagísticos e pragas de insetos. Chemistry Research Journal , v. 1, n. 1, p. 50-57, 2016.

SAWICKA, B.; EGBUNA, C. Pests of Agricultural Crops and Control Measures. In: Natural Remedies for Pest, Disease and Weed Control. Academic Press, 2020. p. 1-16.

SENAPATI, A. et al. Impact of adult mosquito control on dengue prevalence in a multipatch setting: A case study in Kolkata (2014-2015). Journal of theoretical biology, v. 478, p. 139- 152, 2019.

SONAWANE, S. H. et al. Current overview of encapsulation. In: SONAWANE, H. S.; BHANVASE, B. A; SIVAKUMAR, S. Encapsulation of Active Molecules and Their Delivery System. India: Elsevier, 2020. cap. 1, p. 1-8.

SOUSA, S. Chemistry and Toxicology Behind Insecticides and Herbicides. In: RAKHIMOL, K. R. Controlled Release of Pesticides for Sustainable Agriculture. 1. ed. Switzerland: Springer, 2020. Cap. 3, p. 59-109.

SPARKS, T. C. et al. Insecticides, biologics and nematicides: Updates to IRAC's mode of action classification-a tool for resistance management. Pesticide Biochemistry and Physiology, p. 104587, 2020.

SUAVE, J. et al. Microencapsulação: Inovação em diferentes áreas. Revista Saúde e Ambiente/Health and Environment Journal, v. 7, n. 2, p. 12-20, 2006.

SUBBANNA, A. R. N. S. et al. Bio-efficacy of chitinolytic Bacillus thuringiensis isolates native to northwestern Indian Himalayas and their synergistic toxicity with selected insecticides. Pesticide biochemistry and physiology, v. 158, p. 166-174, 2019.

SUGANYA, V.; ANURADHA, V. Microencapsulation and nanoencapsulation: a review. International Journal of Pharmaceutical and Clinical Research, v. 9, n. 3, p. 233-239, 2017.

SUGANYA, V.; ANURADHA, V. Microencapsulation and nanoencapsulation: a review. International Journal of Pharmaceutical and Clinical Research, v. 9, n. 3, p. 233-239, 2017.

TIMILSENA, Y. P. et al. Complex coacervation: Principles, mechanisms and applications in microencapsulation. International journal of biological macromolecules, v. 121, p. 12761286, 2019.

WATANABE, E. et al. Organic solvent-saving sample preparation for systematic residue analysis of neonicotinoid insecticides in agricultural products using liquid chromatographydiode array detection. Food analytical methods, v. 9, n. 1, p. 245-254, 2016.

WOJCIECHOWSKA, M.; STEPNOWSKI, P.; GOŁĘBIOWSKI, M. The use of insecticides to control insect pests. Invertebrate Survival Journal, v. 13, n. 1, p. 210-220, 2016. 
WORLD HEALTH ORGANIZATION. The WHO Recommended Classification of Pesticides by Hazard and Guidelines to Classification 2019. Geneva: World Health Organization, 2020.

YIN, Y.; CADWALLADER, K. R. Spray-chilling encapsulation of 2-acetyl-1-pyrroline zinc chloride complex using hydrophobic materials: Feasibility and characterization of microcapsules. Food chemistry, v. 265, p. 173-181, 2018.

YUSOP, F. H. M.; ABD M. S. F.; HAMZAH, F. Preservation of bioactive compound via microencapsulation. Chemical Engineering Research Bulletin, p. 50-56, 2017.

ZHANG, C. et al. Survey of Asymptomatic Malaria and Mosquito Vectors in Muang Khua District of Phongsaly Province, China-Laos Border. International Journal of Infectious Diseases, 2020.

ZHANG, M. et al. One-step microencapsulation and spraying of pesticide formulations for improved adhesion and sustained release. Journal of microencapsulation, v. 36, n. 7, p. 649- 658, 2019. 